\title{
On the Lifetime of Metastable Metallic Hydrogen
}

\author{
S.N. Burmistrov and L.B. Dubovskii \\ Kurchatov Institute, 123182 Moscow, Russia
}

\begin{abstract}
The molecular phase of hydrogen converts to the atomic metallic phase at high pressures estimated usually as $300-500 \mathrm{GPa}$. We analyze the decay of metallic phase as the pressure is relieved below the transition one. The metallic state is expected to be in the metastable long-lived state down to about $10-20 \mathrm{GPa}$ and decays instantly at the lower pressures. The pressure range of the long-lived metastable state is directly associated with an impossibility to produce a stable hydrogen molecule immersed into the electron liquid of high density. For lower pressures, the nucleation of an electronfree cavity with the energetically favorable hydrogen molecule inside cannot be suppressed with the low ambient pressure.
\end{abstract}

\section{INTRODUCTION}

For the first time, the question on the transition of molecular hydrogen into metallic phase under pressure was apparently attempted by Wigner and Huntington [1] Later, in a great amount of papers [2, 3] (and therein) the equation of state for the metallic state as well as the pressure of the transition into the atomic metallic state are analyzed. In addition, there has arisen a question whether the lifetime of metastable metallic phase could be macroscopically large in some pressure range below the metal-to-molecular phase transition. Here we attempt this problem at zero temperature.

In paper [2] the structure of metallic hydrogen is studied in detail at zero temperature. It is shown in particular that the metallic hydrogen at zero pressure is energetically stable against the decay into separate atoms with the binding energy of about $1 \mathrm{eV}$ per atom. As it concerns the decay into molecules, metallic hydrogen is unstable and the energy of about $2.5 \mathrm{eV}$ releases with escaping a molecule from the metal surface. The escape of hydrogen molecule from the surface of metallic hydrogen should occur via tunneling across a potential barrier. Thus, in general, the lifetime of metallic phase against this process may prove to be sufficiently large. On the other hand, this channel can be withdrawn provided the metallic hydrogen is confined with the corresponding walls.

The formation of molecules is possible not only at the surface of a metal but also in its bulk. The latter process cannot be eliminated. For the formation of a molecule inside the bulk of metallic phase, it is necessary to have a cavity in which the metallic electron density is sufficiently small so that the molecule would be energetically favorable. The inception of a cavity is always associated with increasing the total energy in the system due to extrusion of a metal from the cavity. In order to place a molecule into the cavity, the latter should have a size of several interatomic distances. In general, the energy gain resulted from the formation of a single molecule cannot compensate an increase of the total energy in the system. In any case this occurs in the pressure region near the molecular phase-metal phase transition point since the chemical potentials per atom in the metallic and molecu- lar phases are close to each other. Thus the nucleation of the molecular phase with the large number of molecules in the critical nucleus becomes necessary. The large number of particles in the critical nucleus results inevitably in a drastic reduction of the nucleation probability of such nuclei because this process is a tunneling overcoming of a potential barrier and the tunneling probability depends exponentially on the number of particles.

In the present work we study in what pressure range below the transition pressure the macroscopic description of the nucleus dynamics is possible and how this range depends on the approximations chosen. As we will see later, the lifetime of metallic phase is macroscopically large and practically infinite so long as the macroscopic consideration is possible.

In the opposite case when the critical nucleus is not large and contains a few molecules, the lifetime of the metallic phase is small. This can be estimated as follows. The probability $W_{0}$ for nucleating the critical nucleus as large as a single molecule in a specific site of a bulk is always small

$$
W_{0} \sim \omega_{D} \exp \left(-\alpha \sqrt{m / m_{e}}\right) .
$$

The point is that there is a large factor in the exponent, i.e. square root of a ratio of atom mass $m$ to electron mass $m_{e}$. Here $\omega_{D}$ is a frequency of about Debye frequency in metallic hydrogen $\left(\omega_{D} \sim 10^{14} \mathrm{~s}^{-1}\right.$ and $\alpha$ is the quantity associated with the tunneling motion of hydrogen atoms in the metallic phase in the course of nucleating a molecule. We estimate $\alpha \lesssim 1$ since the typical energy barriers for the motion of nuclei is about $1 \mathrm{eV}$ and distances are of order of $10-20 \mathrm{~nm}$. However, the total probability $W_{\nu}$ is large for the inception of a single nucleus in the bulk containing $\nu \sim 10^{22}$ atoms

$$
W_{\nu} \sim \nu W_{0}
$$

This gives a short lifetime for a metallic hydrogen sample with the large $\nu \sim 10^{22}$ number of atoms.

Note that the same estimate for the small-sized particles of about $\nu \sim 10^{6}$ atoms yields a sufficiently large lifetime. For the process as an escape of molecules from the surface, the lifetime may also prove to be large since $\nu \sim 10^{15}$ in this case. In addition, for the evaporation it 


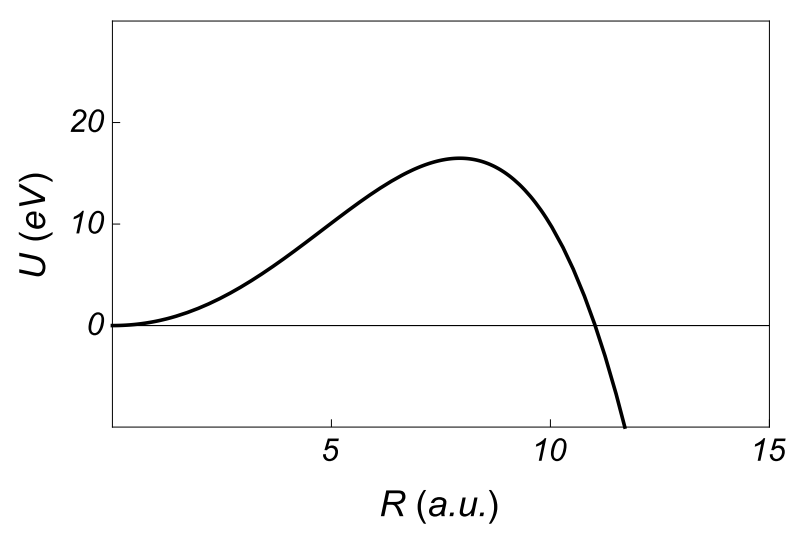

FIG. 1: The potential energy $U$ versus nucleus radius $R$ at ambient pressure $P=40 \mathrm{GPa}$. The critical radius is $R_{c}=11$ a.u. The number of particles in the critical nucleus is $N_{c}=190$.

is essential not the probability of a single event for the formation of a molecule but the evaporation rate determined by escaping the large number of molecules.

To describe a macroscopic nucleus, we employ the Lifshitz-Kagan approach [4]. As a main variable in this approach, we take the density of the phases, i.e., stable (molecular) and metastable (metallic) ones. The potential energy of the system as a function of the nucleus radius $R$ has a typical shape given in Fig. 1. The growth of potential $U$ at small radius $R$ is determined by the effective interphase surface tension and proportional to the radius-squared, i.e. $U(R) \sim R^{2}$ as $R \rightarrow 0$. In the case of the junction between the metallic and molecular phases the effective surface tension is mainly associated with the electron liquid outflow from the metal and with the decrease of the binding energy of a molecule in the electron liquid.

The negativity of potential energy $U$ at large radius $R$ is due to unfavorable difference in energies of metastable metallic and stable molecular phases, i.e. $U(R) \sim-\Delta \mu R^{3}$ where $\Delta \mu$ is a difference in the chemical potentials of the both phases. The transition from metastable state $R=0$ to stable state $R \rightarrow \infty$ occurs via tunneling under potential barrier (Fig. 1) due to kinetic energy $T(R, \dot{R}$ depending on both radius $R(t)$ and growth rate $\dot{R}(t)$

$$
T(R, \dot{R})=M(R) \dot{R}^{2} / 2 .
$$

The mass $M(R)$ in the kinetic energy is associated with a difference in the densities of the metastable and stable phases and results from the outflow or inflow of the substance during the formation of a nucleus.

Besides the various densities the phase transition can be characterized with a number of other internal variables independent of density, e.g. spacing between the nuclei in the course of nucleating a molecule. These internal variables are characterized with the corresponding potential barriers and kinetic energies. Below we suppose that the setting in equilibrium in these variables is the faster process and we take the optimum magnitudes of those variables. In the next section we elucidate the procedure in detail.

As will be shown below, such macroscopic approach, associated with the nucleus dynamics governed with the different phase densities, is possible in a wide range of pressures below the critical one $P_{c} \sim 300-500 \mathrm{GPa}$ down to $10-20 \mathrm{GPa}$. Within this pressure range the critical nuclei have a large number of particles, resulting in a long-lived stability of metallic hydrogen. Note that we underrate the pressure range, neglecting a series of effects which should certainly lead to increasing the lifetime of metallic phase.

\section{PROBLEM STATEMENT}

Let spherical molecular nucleus of radius $R$ be in the metallic hydrogen at the ambient pressure $P$. The potential energy $U(R)$ of a nucleus can be written as (A1)

$$
\begin{gathered}
U(R)=4 \pi \int_{0}^{R} n(r, R)\left(\varepsilon(n(r, R), R-r)-\mu_{0}(P)\right) r^{2} d r \\
+(4 \pi / 3) P R^{3}+4 \pi \sigma R^{2} .
\end{gathered}
$$

Here $\mu_{0}(P)$ is the chemical potential of metallic hydrogen, $n(r, R)$ is the density of the molecular phase at point $r$ of the nucleus with radius $R, \varepsilon(n(r, R), R-r)$ is the energy density of the molecular phase, and $\sigma(P)$ is the surface tension of the interface.

The magnitude of surface tension $\sigma$ and the behavior of energy density $\varepsilon(n(r, R), R-r)$, as a function of the distance from the boundary with the metal, are determined with extending the electron liquid outside the metal into the near-surface region of about Wigner-Seitz radius $r_{s}$ in size [5]. For the energy density $\varepsilon$ of molecular phase, depending on the molecular phase density $n$ and the distance from the metallic hydrogen boundary, we employ simplest approximation

$$
\varepsilon(n, x)=\varepsilon(n)+h(x) .
$$

The first term here corresponds to the energy density of molecular phase for the given density $n$ in the lack of the metal electron density. The second term $h(x)$ implies that the energy of a molecule beside the metal boundary differs significantly from energy $4.7 \mathrm{eV}$ in vacuum taken from the energy of two separate atoms due to dipping a molecule into electron liquid of a metal. The term $h(x)$ can be represented as an external potential affecting the molecule as a result of extending the electron liquid outside the metal into the boundary region of about $r_{s}$ in size. Thus, potential $h(x)$ as well as surface tension $\sigma$ depend on the Wigner-Seitz radius $r_{s}$ or, correspondingly, on the pressure $P$ inside the metal.

In addition, these both quantities, $\sigma$ and $h(x)$, depend on the nucleus size $R$ as well. We will neglect this dependence since we are interested in the macroscopic $R \gg r_{s}$ 
nuclei and the dependence of $\sigma$ and $h(x)$ on radius $R$ becomes insignificant as $R \gtrsim r_{s}$.

Relation (5) corresponds to the gas approximation in the density of molecular phase, meaning a possibility to neglect dependence of $h(x)$ on density $n$. The approximation can be used while the density of molecular phase at the boundary is much smaller than the density of the adjacent metal phase. The point is that potential $h(x)$ is governed with the outflow of electron liquid from the metal, which is almost independent of the strongly localized electron density at the molecule [6, 7]. In the near-surface region, where the magnitude $h(x)$ is large, the density of molecular phase takes the smaller value compared with that at the nucleus center since such density distribution corresponds to the minimum of potential energy $U(R)$. At low $P \lesssim 100 \mathrm{GPa}$ pressures there appears a gap of about $r_{s}$ between the molecular and metallic phases. Inside the gap the density of hydrogen atoms vanishes. For the higher pressures, it is impossible to assert that the density of molecular phase beside the nucleus boundary is much smaller than the density of metallic phase. However, even near the molecular phaseto-metallic phase transition point $P_{c}$ the density of molecular phase differs from that of metallic phase by a factor of 2 . In the next section we discuss function $h(x)$ in detail since this quantity governs mainly the nucleation probability.

Due to the same reason we will neglect the dependence of surface tension $\sigma$ on the molecular phase density at the nucleus boundary, i.e. we put the surface tension equal to its magnitude for the vacuum-metal boundary. For the dependence of the energy density of molecular phase $\varepsilon(n)$ upon $n$, we apply the local approximation $\varepsilon=\varepsilon(n(r, R))$ since the involvement of, e.g., density gradient in $\varepsilon(n)$, corresponds to considering the quantities in such scale which we neglect in describing the potential $h(x)$. This is also associated with the smallness of density gradient in the nucleus due to large nucleus radius $R \gg r_{s}$.

The expression (4) for the potential energy of a nucleus assumes that the internal energy of the system depends on the densities of phases alone. On the whole, this implies the liquid-like description of the both phases. The involvement that the both phase are the crystalline ones increases the potential energy. The liquid-like description of the system means neglecting the shear energy compared with the energy of the bulk compressibility which is completely taken into account in Eq. (4). (See Appendix A.) Such neglect in metallic hydrogen is always justified since the shear modulus is small as compared with the bulk modulus [2, 3]. In molecular hydrogen under low $P \lesssim 10 \mathrm{GPa}$ pressures the both energies, bulk compressibility energy and shear energy, are small compared with the energy of formation of molecules and are inessential in the expression for potential energy. For larger $P \gtrsim 10 \mathrm{GPa}$ pressures, there occurs the same situation as in metallic hydrogen. The shear energy is small compared with the bulk compressibility energy and can be neglected as before.
We will suppose that the nucleus grows slowly, namely, the growth rate $\dot{R}$ of nucleus boundary is much less than the sound velocity $s$

$$
\dot{R} \ll s
$$

In the case of such quasistationary nucleus growth there is a sufficient time to set the mechanical equilibrium in the bulk of the both phases. An existence of mechanical equilibrium in the metastable metallic phase is taken in Eq. (44) into account since the metallic density $n_{0}$ as well as pressure $P$ are assumed to be constant in the derivation of Eq. (4). As it concerns a nucleus, the equilibrium over the nucleus corresponds to constancy of the total chemical potential

$$
\mu(n(r, R))+h(R-r)=C .
$$

Here $C$ is a constant to be determined with the conditions at the nucleus boundary.

Equation (6) can be obtained with varying the potential energy $U(R)$ over nucleus density $n(r, R)$ in (4). On the other hand, this equation is a result of the hydrodynamical Euler equation [8]

$$
\frac{\partial \boldsymbol{v}}{\partial t}+(\boldsymbol{v} \nabla) \boldsymbol{v}=-\frac{1}{n} \nabla P-\nabla h .
$$

Putting $\boldsymbol{v} \rightarrow 0$, we arrive at Eq. (6) since $\nabla P / n=\nabla(\varepsilon+$ $n \partial \varepsilon / \partial n)$ resulted from relation $P=n^{2} \partial \varepsilon / \partial n$. Besides Eq. (6) the minimum in energy $U$ can also be associated with $n \equiv 0$ in some region of a nucleus due to condition $n \geqslant 0$.

Equation (6) and $n=0$ allow us to determine the density distribution $n(r, R)$ in the nucleus bulk. Constant $C$ in (6) can be found using the condition of mechanical equilibrium between the nucleus and the metastable phase. The condition of mechanical equilibrium between the phases is obtained with varying the potential energy $U$ in the nucleus radius $R$ under invariant total number $N$ of particles in the nucleus

$$
\begin{gathered}
(\partial U / \partial R)_{N}=0, \\
N=4 \pi \int_{0}^{R} n(r, R) r^{2} d r .
\end{gathered}
$$

Equation (8) means the following. As the nucleus radius varies, the system keeps the state of the minimum potential energy but has no sufficient time to perform the transition of particles from one phase to the other. Emphasize that this condition holds due to assumption about the quasistationary nucleus growth when the growth rate is small compared with the sound velocity $s$. The latter velocity characterizes the rate of setting the mechanical equilibrium. Equation (8) determines the relation between constant $C(\underline{6})$ and pressure $P$ in the metallic phase (Appendix $\mathrm{B}$ )

$$
\begin{aligned}
P=- & \frac{2 \sigma(P)}{R}-\frac{1}{R^{2}} \int_{0}^{R} n(r) h^{\prime}(R-r) r^{2} d r \\
& +n(R)[C-\varepsilon(n(R))-h(0)] .
\end{aligned}
$$


Here $n(r)=n(r, R)$ and $n(R)=n(R, R)$.

Equations (6), (10) and $n=0$ determine the density distribution $n(r, R)$ in the nucleus at the ambient pressure $P$. If we substitute the above distribution into Eqs. (4) and (9), we obtain the dependence of energy $U$ and particle number $N$ upon nucleus radius $R$. The typical behavior of energy $U$ as a function of radius $R$ is given in Fig. 1. The point $R_{c}$ at which $U\left(R_{c}\right)=0$ corresponds to the critical nucleus radius.

The role of temperature in the quantum transition from the metastable phase with $R=0$ to the state of overcritical nucleus with $R>R_{c}$ is replaced with the kinetic energy in variables $R$ and $\dot{R}$ resulting from the different densities of the phases and outflow of a matter from the nucleus [4]. The kinetic energy of a nucleus is given by

$$
\begin{gathered}
T=T_{1}+T_{2}, \\
T_{1}=\frac{m}{2} 4 \pi \int_{0}^{R} n(r, R) v^{2}(r) r^{2} d r, \\
T_{2}=\frac{m}{2} 4 \pi \int_{R}^{\infty} n_{0}(P) v_{0}^{2}(r) r^{2} d r .
\end{gathered}
$$

Here $m$ is the hydrogen atom mass, kinetic energy $T_{1}$ is associated with the motion of particles in the nucleus, and $T_{2}$ is due to the motion of the mass in the metallic phase. Velocity $v(r)$ inside the nucleus is determined by the continuity equation

$$
\frac{\partial n(r, R)}{\partial t}+\frac{1}{r^{2}} \frac{\partial}{\partial r}\left(r^{2} v(r) n(r, R)\right)=0 .
$$

Remind that density $n$ depends on time $t$ via variable $R=R(t)$ alone and $r$ is a running coordinate. Thus, we have

$$
\frac{\partial n}{\partial t}=\frac{\partial n}{\partial R} \dot{R}
$$

The solution of the above two equations for velocity $v(r)$ at a given distribution $n(r, R)$ can be written as

$$
v(r)=\frac{\dot{R}}{r^{2} n(r, R)} \int_{0}^{r} \frac{\partial n\left(r^{\prime}, R\right)}{\partial R} r^{\prime 2} d r^{\prime} .
$$

The substitution of Eq. (14) into (7) shows that the left-hand side of Eq. (17) is small on the scale of a ratio $\dot{R} / s \ll 1$. So, we have a quasistationary growth of a nucleus and, at first, we can find distribution $n(r, R)$ obeying (6). Then, we substitute the distribution obtained into Eq. (14) to find the velocity distribution $v(r)$ and avoid the combined solution of the Euler equation (7) and continuity equation (12). Substituting (14) into the equation for $T_{1}$ yields

$$
T_{1}=\frac{m}{2} 4 \pi \dot{R}^{2} \int_{0}^{R} \frac{d r}{r^{2} n(r, R)}\left(\int_{0}^{r} \frac{\partial n\left(r^{\prime}, R\right)}{\partial R} r^{\prime 2} d r\right)^{2} .
$$

The velocity distribution $v_{0}(r)$ in the metastable phase obeys the continuity equation (12) as well. However, unlike (13) the term with the time derivative of the density vanishes since the density in the metastable phase is constant for all $R$ and is determined with the ambient pressure $P$. This entails the following behavior [4]

$$
v_{0}(r)=A \dot{R} R^{2} / r^{2} .
$$

The dimensionless factor $A$ can be found using the condition of conserving the total number of particles in the system

$$
d N / d t=4 \pi R^{2} n_{0}(P)\left(\dot{R}-v_{0}(R)\right) .
$$

The left-hand side equals the rate of varying the particle number $N$ in a nucleus and the right-hand side does the incoming flow of particles from the metastable phase. Since the time dependence in (9) enters via variable $R$, we arrive at

$$
\frac{d N}{d t}=4 \pi R^{2} n(R) \dot{R}+4 \pi \dot{R} \int_{0}^{R} \frac{\partial n(r, R)}{\partial R} r^{2} d r .
$$

Using Eqs. (15) - (17), we obtain for $A$

$$
A=1-\frac{n(R)}{n_{0}(P}-\frac{1}{R^{2} n_{0}(P)} \int_{0}^{R} \frac{\partial n(r, R)}{\partial R} r^{2} d r .
$$

Putting Eq. (15) into the expression for $T_{2}$ and calculating the integral in $r$, we represent kinetic energy $T$ in (11) as

$$
\begin{gathered}
T=M(R) \dot{R}^{2} / 2 \\
M(R)=4 \pi m\left[n _ { 0 } ( P ) R ^ { 3 } \left(1-\frac{n(R)}{n_{0}(P)}\right.\right. \\
\left.-\frac{1}{R^{2} n_{0}(P)} \int_{0}^{R} \frac{\partial n(r, R)}{\partial R} r^{2} d r\right)^{2} \\
\left.+\int_{0}^{R} \frac{d r}{r^{2} n(r, R)}\left(\int_{0}^{r} \frac{\partial n\left(r^{\prime}, R\right)}{\partial R} r^{\prime 2} d r^{\prime}\right)^{2}\right] .
\end{gathered}
$$

This expression differs from the corresponding one in [4] because the compressibility of the stable phase is taken into account. In our case the involvement of compressibility is essential since the density of molecular hydrogen varies by a factor of 10 from zero pressure to the $100 \mathrm{GPa}$ pressure region.

The next analysis of nucleation kinetics is based on the hamiltonian with the potential energy (4) and kinetic energy (19). The density distribution in the nucleus bulk is governed with Eqs. (6) and $n=0$ and related with the ambient pressure via Eq. (10).

Deriving the hamiltonian, we have assumed that the state of the system is completely determined with the densities of the phases and the other physical quantities are adjusted adiabatically and unambiguously to the magnitudes of the densities. As an example of such quantities, we can mention the spacing between two atoms in the hydrogen molecule, symmetry of the crystalline lattice in the both phases, and electron density tracing adiabatically the nuclear motion. The adiabatical adjustment of these quantities supposes the slow and quasistationary 
nucleus growth when the setting of all processes in equilibrium occurs faster than the nucleus growth, i.e., transition of particles from the metallic phase to the molecular one. In particular, this implies the mechanical equilibrium between phases (8). The adiabatical relaxation of these parameters means the neglect of contributions of these parameters both to the potential and to the kinetic energies. Such optimization underrates the lifetime of the metastable phase. Setting the equilibrium in the these parameters occurs at about sound velocity or faster as, for example, in the case of adiabatic relaxation of electrons to the motion of nuclei. Hence, we suppose the smallness of nucleus growth rate $\dot{R}$ compared with the sound velocity $s$. Adiabaticity and relaxation of all parameters are equivalent to the fact that the frequency of oscillations, associated with the underbarrier motion, is much smaller than all other frequencies in the system. The frequency $\omega_{b}$, determining the nucleus underbarrier evolution, reduces as the nucleus radius $R_{c}$ grows

$$
\omega_{b} \sim\left(\frac{U_{\max }}{M\left(R_{c}\right) R_{c}^{2}}\right)^{1 / 2} \sim \omega_{D}\left(\frac{r_{s}}{R_{c}}\right)^{3 / 2} .
$$

Here $U_{\max } \sim \sigma R_{c}^{2}$ and $M\left(R_{c}\right) \sim m n_{0} R_{c}^{3}$ where $m$ is an atom mass and $\omega_{D}$ is of the order of the Debye frequency. For the nucleus of large radius $R_{c}$, frequency $\omega_{b}$ is small, entailing a correctness of the quasistationary application. In the case when the size of the critical nucleus is about several interatomic distances it is necessary to take the lack of quasistationary approximation into account.

\section{DISCUSSION OF THE PARAMETERS IN THE HAMILTONIAN}

The potential energy $U(R)$ (4) and kinetic energy $T(R, \dot{R})(19)$ are governed with the following parameters such as: $(i)$ chemical potential of metallic phase $\mu_{0}(P)$ depending on the pressure, (ii) energy $\varepsilon(n)$ and chemical potential $\mu(n)$ depending on the density, (iii) energy variation $h(x)$ of a molecule beside the metal surface, and (iv) surface tension $\sigma(P)$ of a metal. Below we consider the consistent description of the above quantities.

The behavior of energy and chemical potentials as a function of pressure are given for the molecular and metallic phases in Fig. 2. Figure 3 plots the equations of state for the both phases.

We take the results for the metallic phase after [ $\underline{3}$. For the molecular phases, we use the results from the same work [3] and also after [9]. Note that the functions given for metallic hydrogen are obtained with high accuracy at high $P \gtrsim 100 \mathrm{GPa}$ pressures and, correspondingly, at small $r_{s}<1.45$. In zero pressure region of $r_{s} \approx 1.7$ the accuracy of the quantities calculated is smaller and the error for the equation of state within this range may be estimated as $\pm 5 \mathrm{GPa}$.

On the contrary, the equation of state for the molecular phase is well-known in the low pressure region as

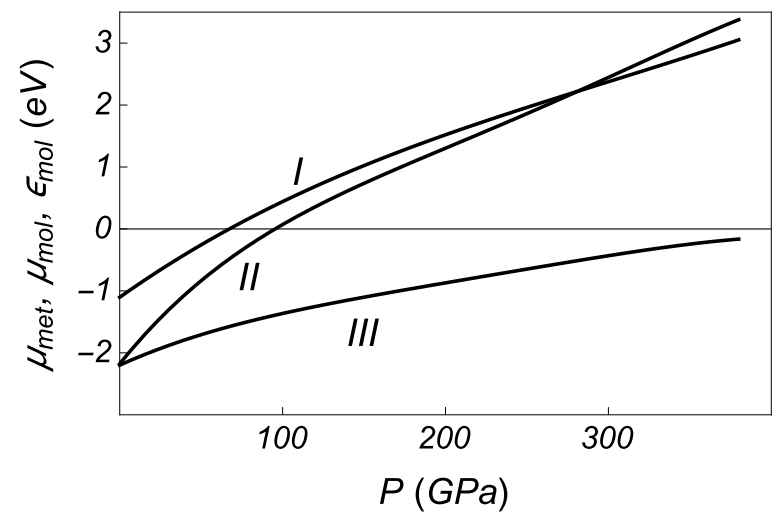

FIG. 2: The chemical potentials of metallic $(I)$ and molecular (II) phases as a function of pressure. Curve $I I I$ is the energy of molecular phase.

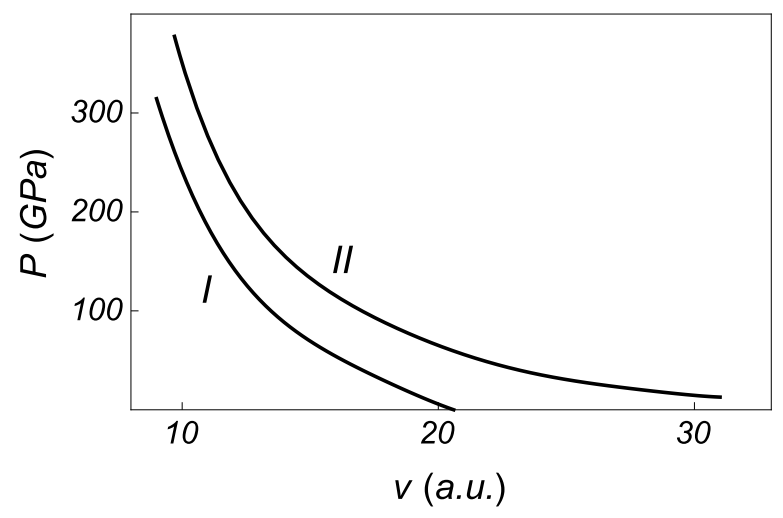

FIG. 3: The equations of state for metallic (I) and molecular (II) phases.

$P \lesssim 10 \mathrm{GPa}$. This is associated with the existence of precise hydrostatic measurements and with the relatively exact description since it is sufficient mainly to consider the pair interactions alone. For higher $P \gtrsim 10 \mathrm{GPa}$ pressures, the consideration of pair interactions alone becomes insufficient [9] and the theoretical description of the equation of state has a worse accuracy. The same is referred to the experiments in this region. An uncertainty in the data for the equation of state results in a dispersion of the phase transition pressure $P_{c}$ [3]. However, for our purposes such dispersion has no principal meaning since the pressure region where the metallic phase exists practically the infinite time is wide and extends down to pressures of about $10 \mathrm{GPa}$. An uncertainty of the equations of states for the phases shifts the pressure boundary by $\pm 5 \mathrm{GPa}$ as the transition pressure of about $300-500 \mathrm{GPa}$. Note that the boundary of the pressure region where the metastable metal exists in the long-lived state is drastic since the lifetime depends on the pressure via large exponent $\beta$. This entails that the small variations of the exponent results in a strong variation of the 


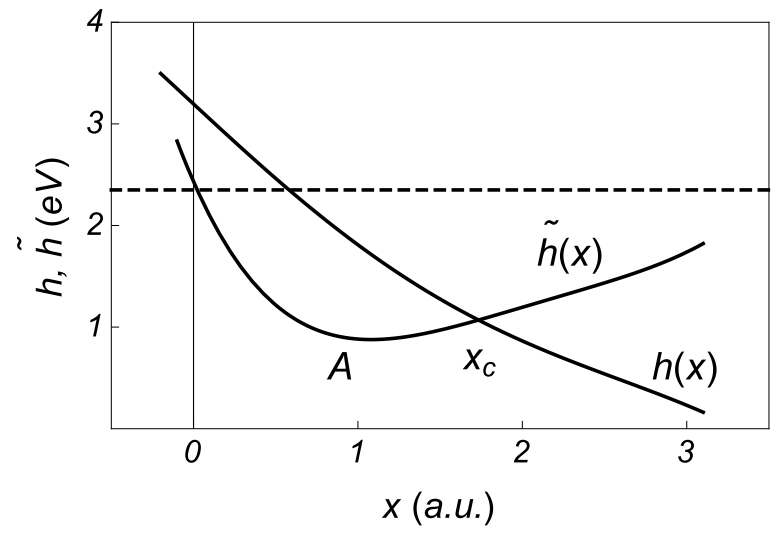

FIG. 4: The functions $h(x)$ and $\tilde{h}(x)$ as a function of distance from the metal surface. The metallic phase is approximated with the jellium model of $r_{s}=1.7$. The dash line corresponds to the energy of a hydrogen atom far from the metal surface.

exponential expression.

The main parameter, which determines the region of the long-lived metastable state, is the energy of a molecule $h(x)$ varying beside the metal surface due to spreading the electron density outside the metal. The magnitude $h(x)$ can be obtained with the direct calculation of the behavior of the energy of a molecule as a function of the distance taken from the metal surface. Such calculation is performed in [6] using the model of jellium. Figure 4 shows the dependence $h(x)$ extrapolated from the data after [6] to $r_{s}=1.7$ corresponding to that of metallic hydrogen at zero pressure [2]. In this figure we also show the energy of a separate hydrogen atom beside the metal jellium surface with $r_{s}=1.7$ denoted as $\tilde{h}(x)$.

Minimum $A$ at the curve $\tilde{h}(x)$ (Fig. (4) corresponds to the energy position of the chemical potential for atoms in metallic hydrogen. At this point there occurs a chemical sorption of hydrogen molecule at the metallic hydrogen surface. This means a possibility of adding new layer to the metallic hydrogen surface. Minimum $A$ of curve $h(x)$ lies higher than the magnitude $h(x)$ at $x \rightarrow \infty$. Otherwise, there occurs an associative chemical sorption at the surface, i.e. chemical sorption with releasing the energy at the transition of a particle from the surface to the infinity. If atoms are located far from the surface as compared with the intersection point $x_{c}$ of curves $h(x)$ and $\tilde{h}(x)$ (Fig. 4), the existence of a molecule becomes possible. For the distances closer than $x_{c}$, the separate atoms are more energetically favorable.

The energy $h(x)$ of a molecule in the field of electron liquid can be subdivided into the energy of atoms in the molecule and the binding energy of a molecule

$$
h(x)=\frac{1}{2}\left[\tilde{h}\left(x-R_{0} / 2\right)+\tilde{h}\left(x+R_{0} / 2\right)\right]+H .
$$

Here $R_{0}$ is the equilibrium distance between the nuclei in the molecule. The binding energy $H$ of a molecule 7] proves to be slightly affected with the orientation of a molecule. Thus, the local approximation governed by the electron liquid density $\rho(x)$ is well adequate, i.e.,

$$
H=h(\rho(x)) \text {. }
$$

The behavior $H(\rho)$, as a function of $r_{s}$, is shown in Fig. [5 and $\rho^{-1}=4 \pi r_{s}^{3} / 3$. The different curves in Fig. 5 correspond to various methods of calculating the function $H$.

Curves 1,2 and 3 are obtained from the data [6] on the behavior of a hydrogen molecule beside the surface of metallic jellium with various $r_{s 0}$ of a metal, namely, 2.07, 2.65 and 4 . Subscript 0 in $r_{s 0}$ differs $r_{s 0}$ of metallic substrate from $r_{s}$ determining the magnitude of electron density with the aid of $\rho^{-1}=4 \pi r_{s}^{3} / 3$. One can see that the curves, obtained with the different ways, are very close to each other and one may say about the universal behavior.

In order to derive the behavior of energy of a molecule as a function of the distance from the metal surface if one knows behavior $H(\rho)$, it is sufficient to apply the behavior $\rho(x)$ beside the surface [5]. The latter problem is one-dimensional and, therefore, is simpler.

One can see from Fig. 5 that the binding energy of a molecule vanishes at the electron density $r_{s}=4.6$. For smaller $r_{s}$, molecule is energetically unfavorable and dissociates into atoms.

Note that the behavior of $h(x)$ and $\tilde{h}(x)$ in Fig. 4 correlates well with the data 2] on metallic hydrogen. The asymptotic behavior $h(x)$ for $x \rightarrow \infty$ is determined with the binding energy of hydrogen molecule and the position of minimum $A$ is governed by the binding energy of an atom in metallic hydrogen. The accuracy of coincidence between the minimum at curve $\tilde{h}(x)$ and the position of the chemical potential for atoms in metallic hydrogen is determined by the neglect of the coupling between atoms in the surface layer. This approximation is analogous to neglecting the dependence $h(x)$ on the density of molecular phase $n$.

While obtaining the plots given in Figs. 4 and 5 , the genuine metal with the discrete structure is replaced with the jellium model. This approximation is well justified at the large distances from the metal. In essence, if $x>r_{s}$, the discreteness of the crystal lattice becomes insignificant. In addition, function $h(x)$ at such large distances is of most interest. The point is that in the range of relatively low pressures of about $10 \mathrm{GPa}$, there appears a spacing between the metal and molecular phases in which the density of molecular phase vanishes. Thus, for such pressures an uncertainty due to inaccurate determination of function $h(x)$ at small distances is negligible. For higher pressures, the lifetime grows more and an additional specifying $h(x)$ in this range becomes inessential. The growth of $h(x)$ at small $x<r_{s}$ distances in the metal with the discrete lattice is reduced as compared with the jellium model since the discrete ions are located from the molecule farther on than for the smoothed background of the jellium. The discrete ions attract electrons stronger and, therefore, electron liquid spreads at smaller distance 


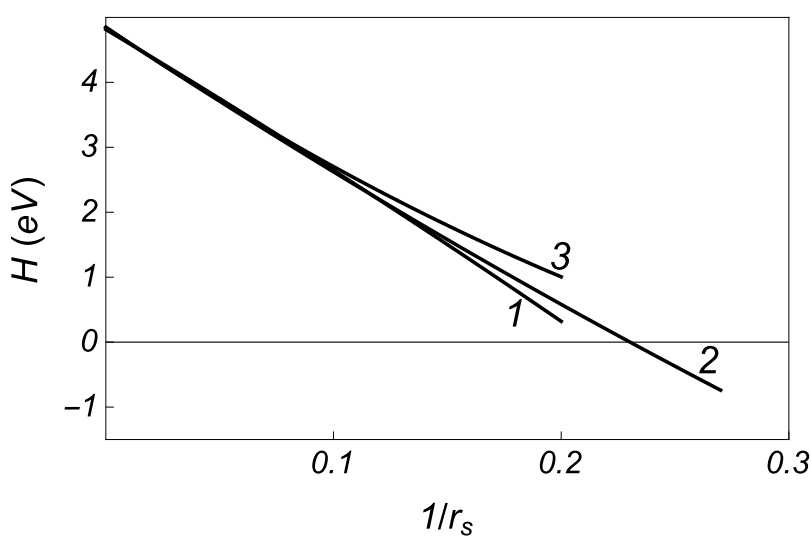

FIG. 5: The plot of binding energy $H$ for a molecule immersed into an electron liquid.

from the surface as compared with the jellium model. This results in the slower enhancement of function $h(x)$ in the metal with the discrete lattice than that in the jellium model. Below we take this fact into account and vary function $h(x)$ at small distances in order to clarify its effect on the lifetime of the metallic phase.

While calculating $h(x)$ in [6], the axis of a molecule is assumed to be normal to the metal surface. Provided one neglects the effect of the molecule orientation on the distribution of electron liquid beside the metal surface and takes into account that the binding energy $H$ of a molecule depends only on the magnitude of the electron liquid density and is independent of the molecule orientation, one can obtain information about $h(x)$ for an arbitrary orientation using the data on $h(x)$ for the normalto-surface orientation of a molecule. For example, in the case when the molecule axis is parallel to the surface the following relation is valid

$$
h_{\|}(x)=\tilde{h}(x)+H(x) .
$$

In the calculation [6] of function $h(x)$ the parameter $R_{0}$, distance between the nuclei in the molecule, keeps unvaried as the molecule approaches the metal surface. This approximation is well justified due to slight dependence of the binding energy on $R_{0}$.

To conclude the discussion of functions $h(x)$ and $\tilde{h}(x)$, we note a few aspects. First, the binding energy of a molecule is positive starting from the electron densities with $r_{s}=4.6$. For lower $r_{s}$, molecule is energetically unfavorable. This specific magnitude $r_{s}$ is three times as larger than $r_{s}$ of metallic hydrogen. Correspondingly, the electron liquid density at the center of cavity, in which the molecule could be placed, should be at least $\approx 30$ times as smaller if compared with the electron density of metallic hydrogen. Thus, to nucleate a single molecule inside the metallic phase, it is necessary to produce a large cavity with the radius of a few $r_{s}$. This fact correlates with the assumption in the previous sections that the nucleation of the molecular phase requires the outflow of a matter in the metallic phase and that the radius of the critical nucleus should significantly exceed $r_{s}$.

The next point to be mentioned is that the hydrogen atom escaping from the metallic phase and traveling from point A (Fig. 4) along curves $h(x)$ and $\tilde{h}(x)$ should overcome an energy barrier. In our model (Sec. III) we neglect a possibility for reflection of hydrogen atom from the energy barrier in the course of quantum tunneling. This implies that we underrate the lifetime of the metastable metallic phase.

Here we emphasize also that the real crossover between the curves $h(x)$ and $\tilde{h}(x)$ is smooth-like since an additional parameter $R_{0}$, distance between the nuclei in the molecule, varies with the distance from the metal surface. In principle, there are possible two situations for the transition from the molecular to metallic phase.

First, $R_{0}$ varies smoothly from the typical spacing between the nuclei in a molecule to that in the metallic phase. Second, variation $R_{0}$ is a jump-like one at the phase interface. Below, as in the previous section, we imply that distance $R_{0}$ follows adiabatically the density. Then a single distinction between these two cases is the following. As distance $R_{0}$ varies continuously, function $h(x)$ behaves more smoothly in the narrow transient region between the phases. So, from this viewpoint it is useful to vary $h(x)$ at distance of about $r_{s}$ in the transient region between the phases. This will be done below with the analysis of the data.

Finally, we discuss the surface tension $\sigma(P)$ of metallic hydrogen for the vacuum-metal boundary. The calculation of the surface tension at the vacuum-metal boundary and comparison with the experimental data has been treated in a large number of works [5], 11] and [12]. In all these papers the consideration is based on the Hohenberg-Kohn-Sham density functional in which the kinetic, exchange and correlation energies of nonuniform electron gas are described as a functional of electron density $\rho(\boldsymbol{r})$. The surface tension of a metal results from the redistribution of electrons and ions beside the metal surface as compared with the bulk distribution. We here employ the simplest version of Ref. 12] when the electron distribution is assumed to be homogeneous in the metal bulk. In this case the surface tension $\sigma$ as a function of pressure is given with curve $I$ in Fig. 6. The curve is well fitted with the relation

$$
\sigma_{I}(P)=-23 P \text { in } \mathrm{erg} / \mathrm{cm}^{2} .
$$

Here pressure $P$ is given in GPa. This approximation neglects a series of contributions to the surface tension. The main contribution neglected is that the density of exchange and correlation energies in nonuniform electron gas has a nonlocal relation with the electron liquid density. Provided this contribution is taken into account as a simple gradient correction, we obtain the surface tensionpressure plot as a curve $I I$ in Fig. 6. The curve is well described with

$$
\sigma_{I I}(P)=-22 P+520 \text { in } \mathrm{erg} / \mathrm{cm}^{2} .
$$




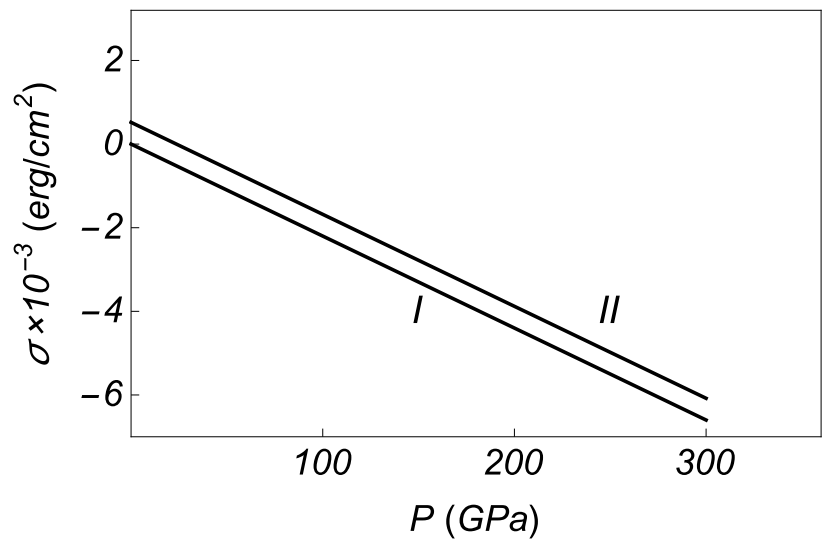

FIG. 6: The surface tension-pressure dependence.

Here we do not discuss the finer effects associated, e.g., with a shift of the edge ion planes beside the metal surface [11] or with nonuniform distribution of electrons in the metal bulk 12] since these contributions are smaller than the term resulted from the gradient exchange-correlation energy. In addition, these corrections become insignificant due to uncertainty of the equation of state in the zero pressure range. Emphasize that the shift of the equation of state with about $5 \mathrm{GPa}$ results in varying the surface tension by about $100 \mathrm{erg} / \mathrm{cm}^{2}$ within the zero pressure range. In what follows, we use mainly expression (24) for the surface tension since this expression results in the stricter condition for the lifetime of the metastable metallic phase.

Note that the final result for the lifetime of metastable metallic hydrogen is not noticeably sensitive whether one takes Eq. (24) or Eq. (25) for the surface tension. The point is that the function $h(x)$ itself results in the effective surface tension which exceeds surface tension $\sigma(P)$ by a few times for the moderate pressures of about $10 \mathrm{GPa}$. For the larger pressures, the effect of surface tension $\sigma(P)$ is weaker due to effect of the bulk term $4 \pi \rho R^{3} / 3$.

\section{QUANTUM NUCLEATION OF THE MOLECULAR PHASE. THE DISCUSSION OF RESULTS}

Like [4], we perform the semiclassical analysis of the tunneling transition between the phases. The classical Lagrangian of the system reads

$$
L(R, \dot{R})=M(R) \dot{R}^{2} / 2-U(R) .
$$

Here effective mass $M(R)$ is determined with Eq. (19) and energy $U(R)$ is given by Eq. (4). In Appendix C the derivative $\partial n(r, R) / \partial R$ in the equation for mass $M(R)$ is transformed to the ambient pressure-fixed expression. The hamiltonian corresponding to the above Lagrangian reads

$$
H=p_{R}^{2} / 2 M(R)+U(R) .
$$

Within our approximation the dynamic description of the system during phase transition is governed with the single principle variable $R=R(t)$ and corresponding momentum $p_{R}$. The initial state of the system is a metastable state. Provided a possibility of the tunneling transition is ignored, the ground state in potential $U(R)$ for radius $R$ close to zero (11) can be estimated with using the uncertainty principle as

$$
p_{R} \cdot R_{\mathrm{typ}} \sim \hbar .
$$

Since radius $R$ is not large, one can approximate potential $U(R)$ as

$$
U(R)=4 \pi R_{\text {typ }}^{2} .
$$

Denoting the ground state energy as $E_{0}=\hbar \omega_{0}$, we have

$$
\omega_{0} \sim 4 \pi \sigma R_{\mathrm{typ}}^{2} / \hbar .
$$

Due to

$$
\frac{p_{R}^{2}}{2 M\left(R_{\mathrm{typ}}\right)} \sim 4 \pi \sigma R_{\mathrm{typ}}^{2}
$$

and using $M(R)=4 \pi m n_{0}(P) R_{\text {typ }}^{3}$, we obtain

$$
R_{\text {typ }} \sim\left[\hbar^{2} /\left(32 \pi^{2} \sigma m n_{0}(P)\right)\right]^{1 / 7}
$$

and

$$
\omega_{0} \sim\left(16 \pi^{3}\right)^{1 / 7} \sigma^{5 / 7} \hbar^{-3 / 7}\left(m n_{0}(P)\right)^{-2 / 7} .
$$

The estimate for $\omega_{0}$ coincides with the semiclassical expression in [4]. Note that unlike $\omega_{b}$ (20), frequency $\omega_{0}$ is independent of critical radius $R_{c}$. The point is that frequency $\omega_{0}$ is associated with the heterophase quantum fluctuations in the homogeneous metastable phase and is insensitive to critical radius $R_{c}$. In the semiclassical approximation the probability for the quantum transition from level $\hbar \omega_{0}$ to nucleation of the critical nucleus is given by

$$
W=\nu \omega_{0} \exp (-\beta), \quad \beta=\frac{2}{\hbar} \int_{0}^{R_{c}}\left|p_{R}\right| d R .
$$

Equation (27) yields

$$
\omega_{0} \sim \omega_{D}\left(m / m_{e}\right)^{3 / 14} \sim 10^{14} s^{-1} .
$$

Here $m / m_{e}$ is a ratio of proton mass to electron one and $\nu$ is the number of virtual nucleation centers of new phase. The latter is of the order of the number of particles in the system. Thus, the preexponential factor coincides with Eqs. (11) and (2) and is about $10^{36}$ particle/s.

Momentum $p_{R}$ is determined semiclassically for the state of energy $E$ close to zero, $\hbar \omega_{0} \ll U_{\max }$, as

$$
\left|p_{R}\right|=\sqrt{2 M(R) U(R)} .
$$




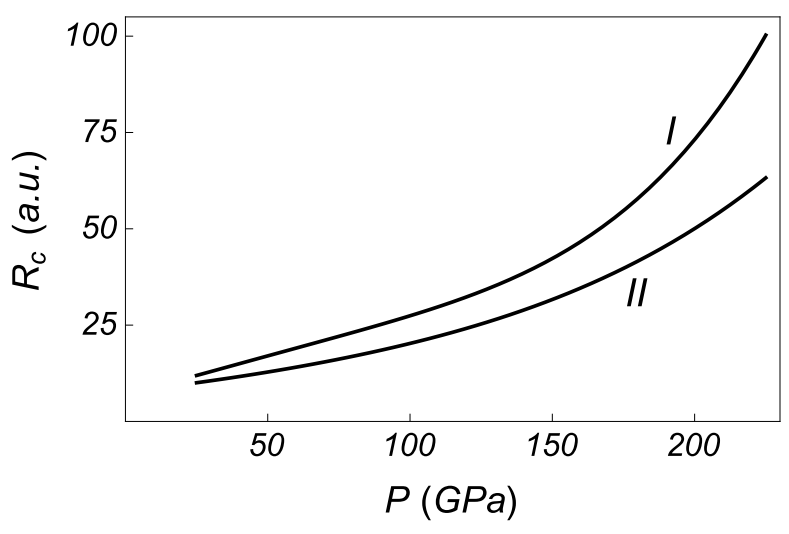

FIG. 7: The critical radius $R_{c}$ versus ambient pressure.

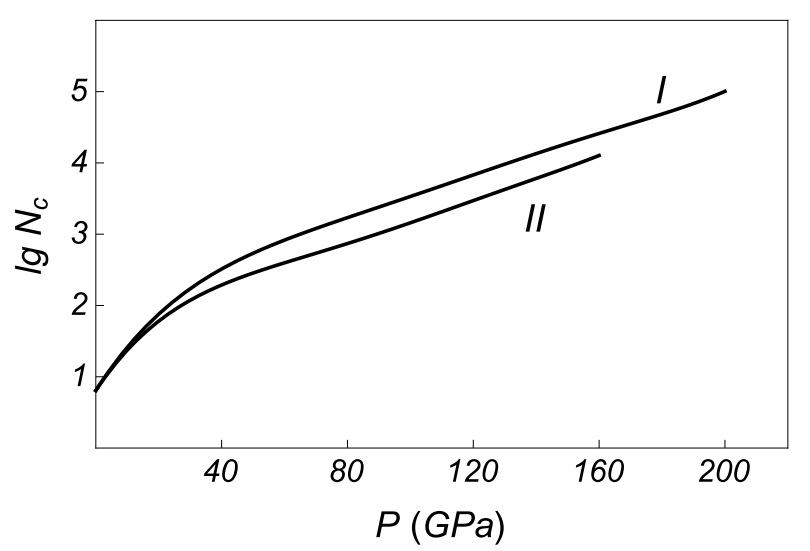

FIG. 8: The number of particles $N_{c}$ in the critical nucleus as a function of ambient pressure (logarithmic scale).

The integration in (28) is performed over the positive region of potential energy $U(R)>0$. To have a macroscopically long-lived state of metastable metallic phase, the exponent $\beta$ in (28) should be large and not smaller than $80-100$. For the smaller exponents, the large preexponential factor in (28) compensates the effect of the exponent, resulting in the large decay probability.

The main parameter determining the exponent is the critical nucleus radius $R_{c}$ or critical number of particles $N_{c}$. The $R_{c^{-}} P$ dependence is plotted in Fig. 7 . Curve $I$ corresponds to choice $h(x)$ obtained by extrapolating the data to metallic hydrogen $r_{s}$ at zero pressure $r_{s}=1.7$ (Fig. 4) and to the surface tension determined with the curve $I$ in Fig. 6. Curve $I I$ in Fig. 7 is obtained with the same surface tension but with the function $h(x)$ changed for $x<1.5$ a.u. Function $h(x)$ is truncated and put equal to $h(x=1.5$ a.u. $)$ (Fig. 4). Such variation $h(x)$ remains the critical radius $R_{c}$ unchanged (Sec. III) at low pressures of about $\lesssim 10 \mathrm{GPa}$ when the region for a long-lived existence of the metastable metallic hydrogen is determined. Thus for determining the boundaries of

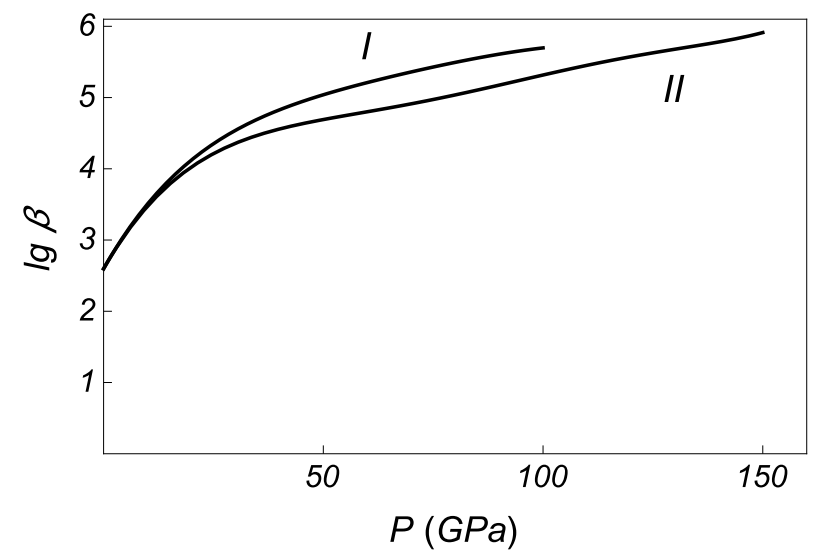

FIG. 9: The exponent $\beta$ (28) versus ambient pressure (logarithmic scale).

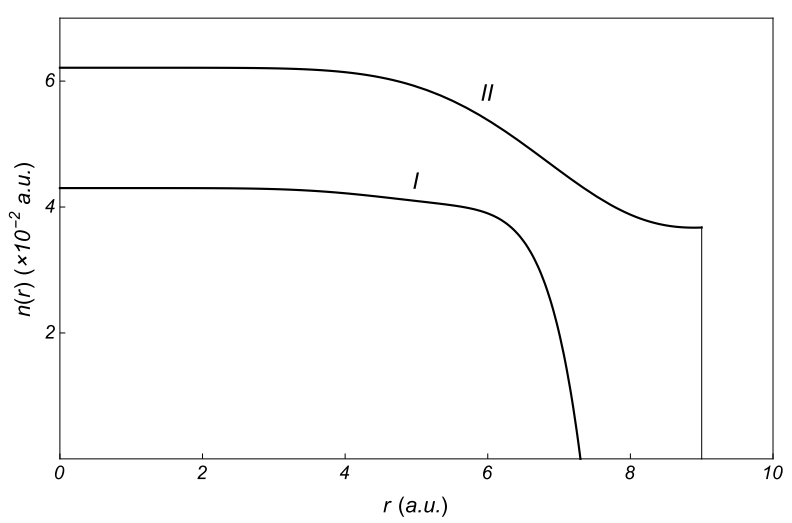

FIG. 10: The density distribution in the nucleus of radius $R=9$ at pressures $20 \mathrm{GPa}$ (curve $I$ ) and $75 \mathrm{GPa}$ (curve $I I$ ).

stability of stable existence of metallic state, the behavior of function $h(x)$ is essential only at large distances from the surface. The behavior at large distances, as noted in Sec. III, is well-known. For the higher pressures when critical radius $R_{c}$ is already large, the truncation mentioned results in some reduction of critical radius $R_{c}$.

In Figs. 8 and 9 we give the plot of the number of particles $N_{c}$ in the critical nucleus and the plot of the exponent $\beta$ in Eq. (28) for different functions $h(x)$. From Figs. 8 and 9 we can obtain the relation between $\beta$ and $N_{c}$. It proves to be that in a wide pressure range this relation can approximately be described with the linear law $\beta=\alpha N_{c}$, $\alpha$ being 200 as $N_{c} \lesssim 100$ and $\alpha$ being 120 as $N_{c} \lesssim 10^{5}$.

In Fig. 10 we plot the typical distribution for the molecular phase density $n(r, R)$ inside the nucleus at various pressures. It is seen that, for the relatively low pressures, there is a spacing $d$ where the density of the molecular phase vanishes. For the pressures larger than $75 \mathrm{GPa}$, the density of molecular phase does not vanish everywhere. 


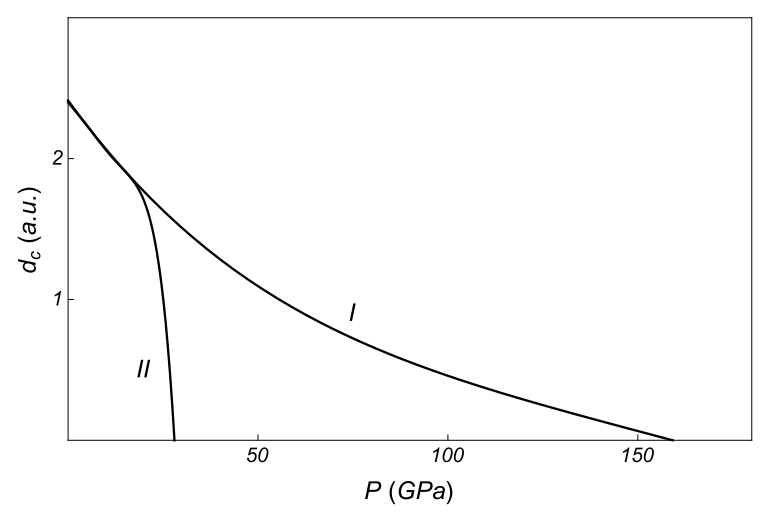

FIG. 11: The spacing $d_{c}=d\left(R_{c}\right)$ for the critical nucleus as a function of pressure for various functions $h(x)$ (curves $I$ and $I I)$.

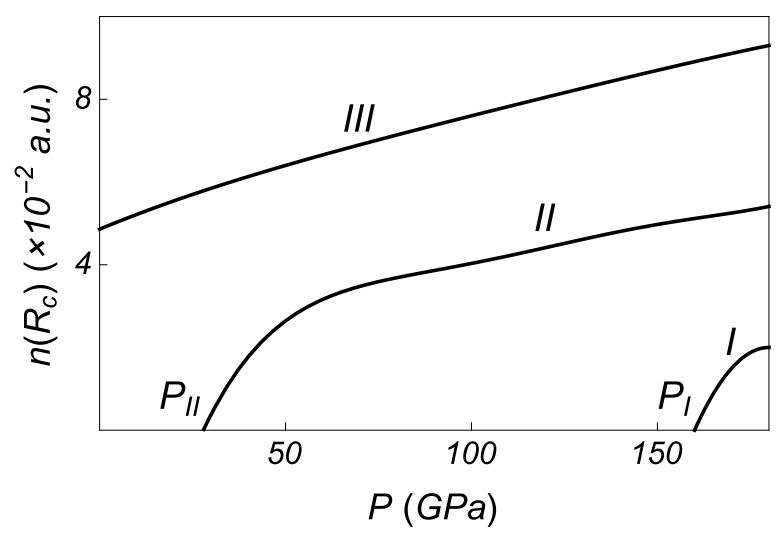

FIG. 12: The dependence of the minimum magnitude of density $n\left(R_{c}\right)$ of molecular phase in the critical nucleus for various functions $h(x)$ (curves $I$ and $I I$ ). The density of metallic phase versus pressure is shown with curve III. For $P<P_{I}, n\left(R_{c}\right)=0$ (curve $I$ ) and for $P<P_{I I}, n\left(R_{c}\right)=0$ (curve $I I$ ).

In Fig. 11 we show the dependence of spacing $d_{c}=d\left(R_{c}\right)$ as a function of pressure $P$ for the critical nucleus.

The plot in Fig. 12]demonstrates the dependence of the minimum magnitude of density $n\left(R_{c}\right)$ of molecular phase in the critical nucleus. Within the whole pressure range the density at the boundary of molecular phase is significantly smaller as compared with the density of metallic phase (curve III in Fig. 12). The latter, as is noted in Sec. III makes it possible to neglect the dependence of function $h(x)$ on the density of molecular phase.

As the data show, the variation of function $h(x)$ within the reasonable limits affects insignificantly the main result, i.e. long-lived stability of existing the metastable metallic phase within the wide pressure range below the transition point $P_{c} \sim 300 \mathrm{GPa}$ down to pressure $\sim 10 \mathrm{GPa}$. The variation of surface tension $\sigma(P)$ affects the results to slight degree. Emphasize that we have used the sur- face tension (Fig. 6, curve $I$ ), resulting in the minimum magnitude of the lifetime.

\section{SUMMARY}

We have analyzed stability of the hydrogen metallic state against nucleation of the stable molecular phase below the transition pressure $P_{c} \sim 300-500 \mathrm{GPa}$. The nucleation dynamics is governed by the tunneling of a critical molecular nucleus through a potential barrier in the low-temperature region and by thermal activation mechanism at high temperatures. In a wide $0.1 P_{c} \lesssim P \leqslant P_{c}$ pressure region below the phase transition pressure $P_{c}$ the critical nucleus of the molecular phase contains a large number of particles and has, correspondingly, a large critical radius as compared with the interatomic spacing. The main reason for the large critical nucleus lies in the impossibility to form a bound state of two hydrogen atoms under high extrinsic electron density of the metallic phase $r_{s} \sim 1.7$. This entails the necessity to produce a cavity inside the metallic phase with the low electron density in the center insomuch that the formation of molecules would become energetically favorable. The nucleation dynamics of molecular nuclei at both low and high temperatures can be described within the framework of the macroscopic approach. Within the mentioned $0.1 P_{c} \lesssim P \leqslant P_{c}$ pressure region the lifetime of the metallic hydrogen phase is macroscopically large and the metallic state is practically stable, i.e. long-lived.

In the low pressure region $P \lesssim 0.1 P_{c}$ the inception of a cavity in the metallic state cannot be suppressed with the applied external pressure $P$ and the critical nucleus amounts to a few particles or less as the external pressure $P$ vanishes. Thus, we expect the opposite behavior with too small lifetime of the metastable metallic state, resulting in practically instant decay of the metallic phase.

\section{Appendix A:}

Let $V_{0}$ and $n_{0}$ be volume and density of the metastable phase. After the nucleation of stable phase of volume $V^{\prime}$ and density $n^{\prime}$ the volume of metastable phase becomes $V$ and density does $n$. The energy of nucleus can be written as

$$
\begin{aligned}
U & =\int_{V} \varepsilon_{0}(n) n d^{3} r+\int_{V^{\prime}} \varepsilon^{\prime}\left(n^{\prime}\right) n^{\prime} d^{3} r \\
& +\int_{V^{\prime}} \sigma d S^{\prime}-\int_{V_{0}} \varepsilon_{0}\left(n_{0}\right) n_{0} d^{3} r .
\end{aligned}
$$

Here $\sigma$ is the surface tension, $\varepsilon_{0}(n)$ and $\varepsilon^{\prime}\left(n^{\prime}\right)$ are the energy density of the metastable and stable phase, respectively. Expanding $\varepsilon_{0}(n)$ in small $\left(n-n_{0}\right)$ as

$$
\varepsilon_{0}(n)=\varepsilon_{0}\left(n_{0}\right)+\left(n-n_{0}\right) P_{0} / n_{0}^{2}
$$


and substituting it into the first equation, we have

$$
\begin{gathered}
U=-\int_{V+V^{\prime}} \varepsilon_{0}\left(n_{0}\right) n_{0} d^{3} r+\int_{V^{\prime}} \varepsilon^{\prime}\left(n^{\prime}\right) n^{\prime} d^{3} r+ \\
\int_{V} \varepsilon_{0}\left(n_{0}\right)\left[n_{0}+\left(n-n_{0}\right)\right] d^{3} r+\frac{P_{0}}{n_{0}} \int_{V}\left(n-n_{0}\right) d^{3} r+\sigma d S^{\prime} \\
=\int_{V^{\prime}} \varepsilon^{\prime}\left(n^{\prime}\right) n^{\prime} d^{3} r-\int_{V^{\prime}} \varepsilon_{0}\left(n_{0}\right) n_{0} d^{3} r \\
+\int_{V}\left(\varepsilon_{0}\left(n_{0}\right)+\frac{P_{0}}{n_{0}}\right)\left(n-n_{0}\right) d^{3} r+\sigma \int_{V^{\prime}} d S^{\prime} .
\end{gathered}
$$

Since

$$
\begin{aligned}
\int_{V}\left(n-n_{0}\right) d^{3} r & =\int_{V} n d^{3} r-\int_{V+V^{\prime}} n_{0} d^{3} r+\int_{V^{\prime}} n_{0} d^{3} r \\
& =\int_{V^{\prime}} n_{0} d^{3} r-\int_{V^{\prime}} n^{\prime} d^{3} r
\end{aligned}
$$

we have finally

$$
U=\int_{V^{\prime}}\left[\varepsilon^{\prime}\left(n^{\prime}\right)-\mu_{0}\left(P_{0}\right)\right] n^{\prime} d^{3} r+P_{0} \int_{V^{\prime}} d^{3} r+\sigma \int_{V^{\prime}} d S^{\prime}
$$

taking $\varepsilon_{0}\left(n_{0}\right)+P_{0} / n_{0}=\mu_{0}\left(P_{0}\right)$ into account.

\section{Appendix B:}

It follows from Eq. (6) that density $n(r, R)$ of molecular phase depends on $C$ as a parameter

$$
n=n(r, R, C) \text {. }
$$

Therefore the potential energy $U$ of a nucleus and the number of particles $N$ depend also on $C$ as a parameter

$$
\begin{aligned}
U & =U(R, C), \\
N & =N(R, C) .
\end{aligned}
$$

Using the last relation, parameter $C$ can be expressed via the total number of particles

$$
C=C(R, N) .
$$

Substituting Eq. (B3) into (B1), we find $U$ as a function of $R$ and $N$

$$
U=U(R, C(R, N))
$$

The condition of mechanical equilibrium (8) reads

$$
\left(\frac{\partial U}{\partial R}\right)_{N}=0
$$

Then we have

$$
\begin{gathered}
4 \pi R^{2}\left(n(R)[\varepsilon(n(R))+h(0)]-\mu_{0}(P) n(R)+P\right) \\
+8 \pi \sigma(P) R+\int_{0}^{R} n(r) h^{\prime}(R-r) 4 \pi r^{2} d r \\
+\int_{0}^{R}\left(\mu(n(r))+h(R-r)-\mu_{0}(P)\right) \\
\times\left[\left(\frac{\partial n}{\partial R}\right)_{C}+\frac{\partial n}{\partial C}\left(\frac{\partial C}{\partial R}\right)_{N}\right] 4 \pi r^{2} d r=0
\end{gathered}
$$

where $n(r) \equiv n(r, R)$ and $n(R)=n(R, R)$. In the last integral the expression in the parentheses is constant due to (6) and can be put in the front of integral. The magnitude of the remaining integral can be found with differentiating Eq. (B2) in $R$

$$
\int_{0}^{R}\left[\left(\frac{\partial n}{\partial R}\right)_{C}+\frac{\partial n}{\partial C}\left(\frac{\partial C}{\partial R}\right)_{N}\right] 4 \pi r^{2} d r+4 \pi R^{2} n(R)=0 .
$$

Then we obtain Eq. (10) for pressure $P$ which can be rewritten in the convenient form for numerics

$$
\begin{gathered}
P=-\frac{2 \sigma(P)}{R}-\frac{1}{R^{2}} \int_{0}^{R}\left[r^{2} n(r)-R^{2} n(R)\right] h^{\prime}(R-r) d r \\
+n(R)[C-\varepsilon(n(R))-h(R)] .
\end{gathered}
$$

\section{Appendix C:}

In the kinetic energy (19) the nucleus mass depends on derivative $\partial n(r, R) / \partial R$, ambient pressure $P$ being fixed. In Eq. (6) the density is directly expressed in terms of $C$ related to pressure $P$. So, it is necessary to transform $\partial n / \partial R$ from one variable to another

$$
\begin{aligned}
& \left(\frac{\partial n}{\partial R}\right)_{P}=\frac{\partial(n, P)}{\partial(R, P)}=\frac{\partial(n, P)}{\partial(R, C)} / \frac{\partial(P, R)}{\partial(C, R)} \\
& =\left[\left(\frac{\partial n}{\partial R}\right)_{C}\left(\frac{\partial P}{\partial C}\right)_{R}-\left(\frac{\partial n}{\partial C}\right)_{R}\left(\frac{\partial P}{\partial R}\right)_{C}\right] /\left(\frac{\partial P}{\partial C}\right)_{R} \\
& =\left(\frac{\partial n}{\partial R}\right)_{C}-\left(\frac{\partial n}{\partial C}\right)_{R}\left(\frac{\partial P}{\partial R}\right)_{C} /\left(\frac{\partial P}{\partial C}\right)_{R} \cdot(\mathrm{C} 1)
\end{aligned}
$$

Differentiating Eq. (6) in $R$ under fixed $C$ and then in $C$ under fixed $R$, we have

$$
\begin{gathered}
\left(\frac{\partial n(r, R)}{\partial R}\right)_{C}=-\frac{h^{\prime}(R-r)}{\mu^{\prime}(n)} \\
\left(\frac{\partial n(r, R)}{\partial C}\right)_{R}=\frac{1}{\mu^{\prime}(n)}
\end{gathered}
$$

Derivatives $(\partial P / \partial R)_{C}$ and $(\partial P / \partial C)_{R}$ are found with differentiating Eq. (10). Though $\sigma$ and $h$ depend on $P$, the derivatives of $\sigma$ and $h$ in $P$ do not enter the ratio $(\partial P / \partial R)_{C} /(\partial P / \partial C)_{R}$. One can see this directly using the cumbersome calculation 


$$
\begin{aligned}
& \frac{(\partial P / \partial R)_{C}}{(\partial P / \partial C)_{R}}=\left\{2 \sigma(P)-R^{2} n(R) h^{\prime}(R)+2 R n(R)[h(R)-h(0)]-\int_{0}^{R}\left[r^{2} n(r)-R^{2} n(R)\right] h^{\prime \prime}(R-r) d r\right. \\
+ & \left.\frac{2}{R} \int_{0}^{R}\left[r^{2} n(r)-R^{2} n(R)\right] h^{\prime}(R-r) d r+\int_{0}^{R} \frac{h^{\prime 2}(R-r)}{\mu^{\prime}(n)} r^{2} d r\right\} \times\left(R^{2} n(R)-\int_{0}^{R} \frac{h^{\prime}(R-r)}{\mu^{\prime}(n(r, R))} r^{2} d r\right)^{-1} .
\end{aligned}
$$

Substituting the above three relations for the derivatives into Eq. (C1), we obtain the relation for $\partial n / \partial R$ which should be employed for calculating $M(R)$ (19) under fixed pressure $P$.
[1] E. Wigner and H. B. Huntington, J. Chem. Phys. 3, 764 (1935).

[2] E. G. Brovman, Yu. Kagan, and A. Kholas, ZhETF 61, 2429 (1971) [Sov. Phys. JETP 34, 1300 (1972)].

[3] Yu. Kagan, V. V. Pushkarev, and A. Kholas, ZhETF 73, 967 (1977) [Sov. Phys. JETP 46, 511 (1977)].

[4] I. M. Lifshitz and Yu. Kagan, ZhETF 62, 385 (1972) [Sov. Phys. JETP 35, 206 (1972)]; S. N. Burmistrov and L. B. Dubovskii, Phys. Lett. A 127, 79 (1988).

[5] N. D. Lang and W. Kohn, Phys. Rev. 1, 4555 (1970).

[6] H. Hjelmberg, B. I. Lundqvist, and J. K. Nørskov, Phys. Scripta 20, 192(1979).
[7] J. K. Nørskov, Phys. Rev. B 20, 446 (1979).

[8] L. D. Landau and E. M. Lifshitz, Fluid Mechanics (Pergamon, Oxford, 1987).

[9] S. I. Anisimov and Yu. V. Petrov, ZhETF 74, 778 (1978) [Sov. Phys. JETP 47, 407 (1972)].

[10] J. K. Nørskov, Solid State Comm. 24, 691 (1977).

[11] M. Hietschold, G. Paasch and P. Ziesche, Phys. Stat. Sol. (b) 70, 653 (1975).

[12] S. N. Burmistrov and L. B. Dubovskii, Fiz. Tverd. Tela 23, 3104 (1981).

[13] V. Sahni and C. Q. Ma, Phys. Rev. B 20, 3511 (1974). 